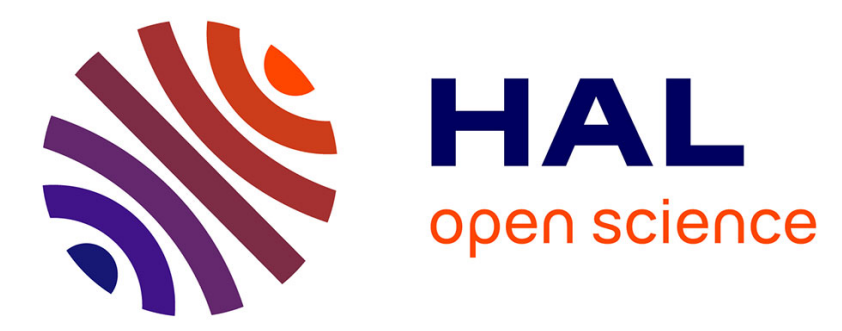

\title{
Glass forming region and optical properties of chalcogenide glasses within a gallium-tin-selenium ternary system
}

J. Zhang, Y. Li, C. Zhang, F. Chen, Xianghua Zhang, W Ji

\section{- To cite this version:}

J. Zhang, Y. Li, C. Zhang, F. Chen, Xianghua Zhang, et al.. Glass forming region and optical properties of chalcogenide glasses within a gallium-tin-selenium ternary system. Journal of NonCrystalline Solids, 2020, 545, pp.120240. 10.1016/j.jnoncrysol.2020.120240 . hal-02892719

\section{HAL Id: hal-02892719 \\ https://hal.science/hal-02892719}

Submitted on 23 Nov 2020

HAL is a multi-disciplinary open access archive for the deposit and dissemination of scientific research documents, whether they are published or not. The documents may come from teaching and research institutions in France or abroad, or from public or private research centers.
L'archive ouverte pluridisciplinaire HAL, est destinée au dépôt et à la diffusion de documents scientifiques de niveau recherche, publiés ou non, émanant des établissements d'enseignement et de recherche français ou étrangers, des laboratoires publics ou privés. 


\title{
Glass forming region and optical properties of chalcogenide
}

\section{glasses within a gallium-tin-selenium ternary system}

Jiao Zhang ${ }^{\text {a, b }}$, Yang Li ${ }^{\text {a, b }}$, Chen Zhang ${ }^{\text {a, b }}$, Feifei Chen ${ }^{\text {a, b, * }}$, Xianghua Zhang ${ }^{c}$, Wei Ji ${ }^{\text {d }}$ ${ }^{a}$ Laboratory of Infrared Materials and Devices, The Research Institute of Advanced Technologies, Ningbo University, Ningbo 315211, China

${ }^{\mathrm{b}}$ Key Laboratory of Photoelectric Detection Materials and Devices of Zhejiang Province, Ningbo University, Ningbo, 315211, China

${ }^{\mathrm{c}}$ Laboratory of Glasses and Ceramics, UMR 6226 CNRS-University of Rennes 1, Rennes Cedex 135042, France

${ }^{\mathrm{d} D e p a r t m e n t}$ of Physics, National University of Singapore, 2 Science Drive 3, 117551,

Singapore

* Corresponding author. E-mail address: chenfeifei1@nbu.edu.cn

\begin{abstract}
In this work, glass forming region of arsenic- and germanium-free chalcogenide glasses (ChGs) within a gallium-tin-selenium (Ga-Sn-Se, GSS) ternary system is depicted. Seven representative compositions in the glass forming region are selected, and the corresponding bulk GSS glass samples are prepared for characterizing the optical properties. The experimental results show that the GSS ChGs have a wide transparent window from $\sim 1$ to $20 \mu \mathrm{m}$ and low dispersion at both 3-5 $\mu \mathrm{m}$ and 8-12 $\mu \mathrm{m}$ mid-infrared windows. The dependence of optical bandgap energy $\left(E_{\mathrm{opg}}\right)$ on mean coordination number $(\mathrm{MCN})$ indicates the existence of medium-range interactions in the GSS glass network, and its origin is investigated by the Raman spectra.
\end{abstract}


Keywords: Selenide chalcogenide glasses; Ga-Sn-Se system; glass forming region; optical properties.

\section{Introduction}

Chalcogenide glasses (ChGs) have the highest third-order optical nonlinearity (TONL) in all optical glass materials, and they also possess ultra-wide infrared (IR) transmission windows as well as low phonon energy, high linear refractive index and low thermal refractive index coefficient, which make ChGs a perfect candidate for both IR and nonlinear photonic devices, especially all-optical switching, optical frequency comb, supercontinuum generation [1-5].

In the past few decades, ChGs based on germanium (Ge)-chalcogen and arsenic (As)-chalcogen systems have been intensively studied for their excellent glass forming ability, wide IR transparent windows and high thermal stability [6-11]. However, considering the high price of Ge and toxicity of As, ChGs in As- and Ge-free systems with high optical performance are also in great demand, but very few studies can be found besides the gallate ChGs based on Ga-La-S ternary system [12-14] despite its preparation process is much more complex than the conventional ChGs. Recently, Yang et al. [15, 16] reported the glass forming ability of gallate sulfide ChGs based on Ga-Sb-S ternary system and found large TONL of the ChGs as well as expanded IR transmittance region compared to Ge- or As-based sulfide ChGs, which gives the society of ChG materials new insight into the development of novel gallate ChGs with similar preparation process but better properties as compared to the conventional ChGs.

On the other hand, considerable recent studies with respect to chalcogenide photonic devices $[17,18]$ have been focused on selenide ChGs which possess wider IR transmittance region and larger TONL properties as compared the sulfide ChGs. 
Additional, our prior studies $[19,20]$ have discovered that the incorporation of tin $(\mathrm{Sn})$ to selenide ChGs could significantly enhance the TONL properties and eliminate impurity absorption. Therefore, it would be an interesting subject to elaborate on $\mathrm{Sn}$ contained gallate selenide ChGs, i.e. gallium-tin-selenium (Ga-Sn-Se, GSS) ternary system glasses, which maybe a green/sustainable and low-cost ChG system comparing with the As- and Ge-based ChGs. However, to the best of our knowledge, ChGs in GSS ternary system remain unexplored, which motivates us to conduct the corresponding research. Accordingly, the goal of this paper is to give the glass forming region of the GSS ternary system as well as the properties of the GSS ChGs, and to elucidate the correlation to their network structure.

\section{Experiment}

A set of chemical compositions in Ga-Sn-Se ternary system were designed for exploration of the corresponding glass forming region, the specific data are given in supplementary materials. $10 \mathrm{~g}$ of analytical reagent gallium, tin and selenium as raw materials were weighed according to the elemental ratio and mixed in a quartz tube $(\Phi 10 \mathrm{~mm})$. The quartz tube containing the raw material was pre-heated at $100{ }^{\circ} \mathrm{C}$ for

$4 \mathrm{~h}$ to remove moisture from the quartz tube and the raw material which was then sealed under vacuum $\left(10^{-4} \mathrm{~Pa}\right)$ and melted in a rocking furnace. The melting temperature of the samples was set at $900{ }^{\circ} \mathrm{C}$ and maintained for 12 hours to ensure sufficient reaction of the raw materials. After that, the tube was rapidly quenched in water to form ingot. For the glass samples applied for further properties testing, the corresponding ingots were annealed at a temperature of $20^{\circ} \mathrm{C}$ below the glass transition temperature $\left(T_{\mathrm{g}}\right)$ for 6 hours to minimize internal stress and slowly cool to room temperature at a rate of $10{ }^{\circ} \mathrm{C} / \mathrm{h}$. For optical properties testing, the glass ingots were then cut and polished to the plate with thickness of $1 \mathrm{~mm} \pm 0.02 \mathrm{~mm}$ which gave 
rise to the main error of $\pm 1 \%$ in calculation of optical bandgap energy $\left(E_{\mathrm{opg}}\right)$ of the glass samples.

To identify nature of the obtained fusions, X-ray diffractometer (D2 phaser, Bruker, Germany) was used, and the samples with sharp diffraction peaks were identified as crystalline. About $10 \mathrm{mg}$ powder was sealed into an aluminum pan for $T_{\mathrm{g}}$ and $T_{\mathrm{x}}$ measurements using a differential scanning calorimeter (DSC, TA Q2000, USA), and the instrumental error is $\pm 1{ }^{\circ} \mathrm{C}$. The thermal stabilit y parameter $\Delta T$ calculated by $T_{\mathrm{g}}$ and $T_{\mathrm{x}}$ has an error of $\pm 2{ }^{\circ} \mathrm{C}$. Transmission spectra of the re-prepared samples in wavelength range from 0.4 to $2.5 \mu \mathrm{m}$ were recorded by a UV-VIS-NIR spectrophotometer (Lamda 950, Perkin-Elmer, Waltham, MA) and wavelength range from $2.5 \mu \mathrm{m}$ to $25 \mu \mathrm{m}$ were recorded by a Fourier transform IR (FTIR) spectrometer (Nicolet381, Nicolet, USA). The samples with flat transmittance above 50\% were identified as glass, otherwise partial crystalline. Authentic molar percentage of each elements in the GSS ChGs was determined by energy-dispersive spectrometry (EDS, XFlash Detector 410-M, Bruker, Germany), and the instrumental error is $\pm 1 \%$. Density (d) was measured using Archimedes method and each $d$ is average of eight measurements with instrumental errors of $\pm 0.001 \mathrm{~g} / \mathrm{cm}^{3}$.

Linear refractive index ( $n_{0}$ in wavelength range from 1.7 to $20 \mu \mathrm{m}$ ) of the GSS ChGs was estimated using an IR variable angle spectroscopy ellipsometer with a precision of \pm 0.005 (IR-VASE Mark II, J.A. Woollam, USA), where the angles of incident were selected $65^{\circ}$ and $75^{\circ}$, respectively, and spectral resolution was $16 \mathrm{~cm}^{-1}$. Thus, the calculated results of partial dispersion $v_{3-5}$ and $v_{8-12}$ had approximately $\pm 1 \%$ error which is three-fold to that of the $n_{0}$ value. Raman spectra of the glasses were recorded using a Renishaw Invia Raman microscope (Renishaw Invia, Gloucestershire, UK) with an excitation wavelength of $785 \mathrm{~nm}$ and frequency 
resolution of $\pm 0.15 \mathrm{~cm}-1$. The calculation error of the proportion of the integral area of each structural unit by Gaussian decomposition of Raman spectrum is about $\pm 5 \%$, which is within acceptable limits.

All the above tests were conducted at room temperature.

\section{Results}

Fig. 1 shows the glass forming region of the GSS ChG system, and nature of the ingots prepared from each molar composition are also given in supplement material. It can be seen from Fig. 1 that the GSS ternary system has a small glass forming region located in Se-rich region which is composed of $\sim 65 \%-80 \% \mathrm{Se}, \sim 5 \%-15 \% \mathrm{Ga}$, and $\sim 15 \%-25 \%$ Sn, meaning that the compositions in both stoichiometry and Sedeficiency are not able to keep the bulk in amorphous state. As presented in Table 1, seven compositions in glass forming region were employed to represent the general properties of ChGs in the GSS system. Fig. 2(a) shows the XRD patterns of the selected seven samples, the absence of sharp diffraction peaks in the pattern clearly illustrates that the selected GSS compositions were amorphous. For comparison, Fig. 2(b) shows the XRD patterns of two compositions ( $\mathrm{Ga}_{10} \mathrm{Sn}_{15} \mathrm{Se}_{75}$ and $\left.\mathrm{Ga}_{5} \mathrm{Sn}_{10} \mathrm{Se}_{85}\right)$ out of the glass forming region, which were identified as partially crystalline by the presence of diffraction peaks belonging to $\mathrm{Ga}_{2} \mathrm{Se}_{3}$ phase (JCPDF No. 5-724).

The measured molar composition of the seven GSS specimens by EDS are given in Table 1, and they are generally in consistent with the nominal ones. The main deviation of the measured composition from the nominal is slight loss of Sn content, that's probably because of the precipitate of Sn atoms that have much larger size and weight than the $\mathrm{Ga}$ and Se atoms. Glass transition temperature $\left(T_{\mathrm{g}}\right)$ and crystallization temperature $\left(T_{\mathrm{x}}\right)$ of the GSS ChGs as given in Table 1. It can be seen that the $T_{\mathrm{g}}$ value increased by over $80{ }^{\circ} \mathrm{C}$ when $\mathrm{Se}$ was replaced by both $\mathrm{Ga}$ and $\mathrm{Sn}$. Since $T_{\mathrm{g}}$ of glass 
materials is determined by average bond energy and connectedness of the glass network [21, 22], substitution of homopolar bonds (Se-Se bond: $183.9 \mathrm{~kJ} / \mathrm{mol}$ ) by heteropolar bonds (Sn-Se bond: $198.5 \mathrm{~kJ} / \mathrm{mol}$, Ga-Se bond: $271.7 \mathrm{~kJ} / \mathrm{mol}$ ) [23, 24] that have larger bond energy is accounted for the increased $T_{\mathrm{g}}$ in ChGs with higher Ga and $\mathrm{Sn}$ content. Besides, calculation of thermal stability parameter $\Delta T=\left(T_{\mathrm{x}}-T_{\mathrm{g}}\right)$ shows that increasing content of $\mathrm{Ga}$ which increased $T_{\mathrm{g}}$ would reduce total thermal stability of the GSS ChGs. Sample GSS1 with lowest Ga content have the largest $\Delta T$, and the value near $150{ }^{\circ} \mathrm{C}$ indicating that this composition is suitable for fiber drawing. Further, as given in Table 1, $T_{\mathrm{g}}$ of the GSS samples (GSS5 7) is comparable to that of the Ge-Sb-Se and Ga-Sb-S ChGs, and much higher than that of the As40 $\mathrm{Se}_{60} \mathrm{ChG}$.

As the optical transmission spectra shown in Fig. 3(a), all the GSS ChGs exhibit flat transmittance window range from $\sim 1$ to $20 \mu \mathrm{m}$, and they are optically transparent even beyond $25 \mu \mathrm{m}$. Since the raw materials were not purified, impurity absorption [25] due to $\mathrm{O}-\mathrm{H}, \mathrm{Se}-\mathrm{H}, \mathrm{H}_{2} \mathrm{O}, \mathrm{Ga}-\mathrm{O}$ and $\mathrm{CO}_{2}$ can be observed at $2.9 \mu \mathrm{m}, 4.5 \mu \mathrm{m}, 6.3$ $\mu \mathrm{m}, 9.5 \mu \mathrm{m}$ and $15 \mu \mathrm{m}$ respectively. No impurity absorption from $\mathrm{Sn}-\mathrm{O}$ is observed since it is beyond $20 \mu \mathrm{m}$ [24] and overlapped by the harmonic intrinsic vibrations of the components. Fig. 3(b) shows the comparison of IR transmittance region of the sample GSS1 and the commercial As40 $\mathrm{Se}_{60}$ and $\mathrm{Ge}_{20} \mathrm{Sb}_{15} \mathrm{Se}_{65}$ selenide ChGs with the same thickness of $1 \mathrm{~mm}$, and it is clear that the GSS ChG possess extended IR transmittance range: the long-wavelength cut-off of sample GSS1 red-shifted by 3.2 $\mu \mathrm{m}$ and $1.3 \mu \mathrm{m}$ compared to that of the $\mathrm{Ge}_{20} \mathrm{Sb}_{15} \mathrm{Se}_{65} \mathrm{ChG}$ (cut-off at $16.5 \mu \mathrm{m}$ ) and $\mathrm{As}_{40} \mathrm{Se}_{60} \mathrm{ChG}$ (cut-off at $18.4 \mu \mathrm{m}$ ) respectively. Furthermore, transmittance of GSS ChGs $(>70 \%)$ is remarkably higher than those of both $\mathrm{As}_{40} \mathrm{Se}_{60}$ and $\mathrm{Ge}_{20} \mathrm{Sb}_{15} \mathrm{Se}_{65}$ ChGs, due to lower reflection loss originated from the smaller refractive index $\left(n_{0}\right.$, given in Table 2) of the GSS ChGs. 
Near IR absorption spectra of the GSS ChGs are shown in Fig. 4. It can be seen that the fundamental absorption edge of sample GSS1 cut-off at $\sim 750 \mathrm{~nm}$, and it shifts towards longer wavelength (red-shifting) with increase of Ga content. As a result, GSS7 with highest Ga content shows the most red-shifted absorption edge. According to the previous studies [26], optical bandgap energy $\left(E_{\mathrm{opg}}\right)$ corresponding to the photon energy of wavelength at linear absorption coefficient is $100 \mathrm{~cm}^{-1}$ can be used to quantitatively analyze the composition dependent shifting of the fundamental absorption edge. As the data listed in Table 2, $E_{\text {opg }}$ value of the GSS ChGs are close to the commercial $\mathrm{As}_{40} \mathrm{Se}_{60}$ and $\mathrm{Ge}_{20} \mathrm{Sb}_{15} \mathrm{Se}_{65} \mathrm{ChGs}$, and composition modification of the GSS ChGs leads to $0.1 \mathrm{eV}$ total variation of the $E_{\text {opg }}$ value.

Linear refractive index $\left(n_{0}\right)$ and the wavelength dependence, namely dispersion curves of the GSS ChGs are presented in Fig. 5. It can be seen that all the curves show a sudden drop from 1.7 to $3 \mu \mathrm{m}$, indicating large dispersion of the GSS ChGs at this wavelength region. In longer wavelength region $(>3 \mu \mathrm{m})$, decrease of $n_{0}$ with wavelength slows down, implicating attenuation of the dispersion property. Sample GSS5 exhibits the largest $n_{0}$ value at all wavelength region, while sample GSS2 has the minimum probably account for its lowest $\mathrm{Sn}$ content. Since low dispersion is highly required to minimize chromatic aberration in photonic devices, evaluation of the MIR dispersion behavior of the GSS ChGs at the two MIR atmospheric windows (3-5 $\mu \mathrm{m}$ and 8-12 $\mu \mathrm{m}$ ) are performed by defining partial dispersion $\nu_{3-5}$ and $v_{8-12}$, and the formula is:

$$
v_{\mathrm{S}-\mathrm{L}}=\begin{gathered}
n_{\mathrm{M}}-1 \\
n_{\mathrm{S}}-n_{\mathrm{L}}
\end{gathered}
$$

in which $\mathrm{S}$ and $\mathrm{L}$ refer to short and long wavelength of interests respectively, and $\mathrm{M}$ is the wavelength in the middle of them. The calculated data are shown in Table 2, most of the GSS ChGs have $v_{3-5}$ value larger than $v_{8-12}$ value, which means that dispersion 
of the ChGs is weaker at the first MIR window. $v_{3-5}$ and $v_{8-12}$ values of GSS5 are the smallest meaning that this sample has the strongest dispersion in MIR account for its largest $n_{0}$; GSS2 has relatively larger $v_{3-5}$ and $v_{8-12}$ value for its smallest $n_{0}$. The $n_{0}$ and partial dispersion values of $\mathrm{Sn}_{10} \mathrm{Sb}_{20} \mathrm{Se}_{70}, \mathrm{As}_{40} \mathrm{Se}_{60}$ and $\mathrm{Ge}_{20} \mathrm{Sb}_{15} \mathrm{Se}_{65}$ ChGs are also given in Table 2 for reference. It can be found that $n_{0}$ values of both $\mathrm{As}_{40} \mathrm{Se}_{60}$ and $\mathrm{Ge}_{20} \mathrm{Sb}_{15} \mathrm{Se}_{65}$ ChGs are larger than those of all the GSS ChGs. GSS2 has comparable dispersion to that of $\mathrm{Ge}_{20} \mathrm{Sb}_{15} \mathrm{Se}_{65} \mathrm{ChG}$ at 3-5 $\mu \mathrm{m}$ window, and dispersion of all GSS ChGs is weaker than that of $\mathrm{Ge}_{20} \mathrm{Sb}_{15} \mathrm{Se}_{65}$ at 8-12 $\mu \mathrm{m}$ window. Compared to the $\mathrm{As}_{40} \mathrm{Se}_{60} \mathrm{ChG}$, all the GSS ChGs exhibit relatively stronger dispersion at 3-5 $\mu \mathrm{m}$ window and 8-12 $\mu \mathrm{m}$ window. Additional, dispersion of the the GSS ChGs is smaller than the $\mathrm{Sn}_{10} \mathrm{Sb}_{20} \mathrm{Se}_{70} \mathrm{ChG}$.

\section{Discussion}

In order to find correlation between molar composition and optical properties of the GSS ChGs, the constraints model using mean coordination number (MCN) as a fundamental metric is applied. According to the authentic molar percentage of each ChG given in Table 1 , the MCN value can be calculated by the following equation [27]:

$$
\mathrm{MCN}=\begin{gathered}
x C N_{\mathrm{Ga}}+y C N_{\mathrm{Sn}}+z C N_{\mathrm{Se}} \\
x+y+z
\end{gathered}
$$

where $x, y$ and $z$ are molar percentage of $\mathrm{Ga}$, Sn and Se respectively, and $C N$ is their coordination number. Since the all the GSS specimens have Se-rich compositions, both $\mathrm{Ga}$ and $\mathrm{Sn}$ atoms would be 4 -fold coordinated $[23,28]$ and connected with the 2-fold coordinated Se atoms. The calculated MCN values are listed in Table 1, it can be seen that the GSS ChGs have MCN range from 2.42 to 2.69 covering a topological threshold at $\mathrm{MCN} \approx 2.6$ known as the starting point of medium-range interactions of 
layered structures in ChG network [29]. By plotting the $E_{\text {opg }}$ of the GSS ChGs as a

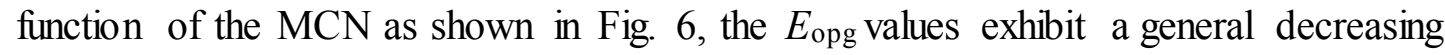
tendency with increase of $\mathrm{MCN}$, while two abnormal points (i.e. rising of $E_{\mathrm{opg}}$ ) at $\mathrm{MCN}=2.55$ and 2.65 can be observed as well. Such behavior can be attributed to the normal structural evolution with molar compositions in combination with the occurrence of medium-range interactions among the structural units in GSS glass network. Therefore, Raman spectra of the seven GSS samples are measured in order to uncover the structural evolution that accounts for the MCN-dependent optical properties as well as the medium-range interactions in GSS ChGs. In general, as the spectra shown in Fig. 7, it can be seen that the GSS ChGs have general Raman signal in frequency range from 150 to $300 \mathrm{~cm}^{-1}$. The main Raman peak located at $\sim 180 \mathrm{~cm}^{-1}$ belongs to vibration mode of $\mathrm{Sn}-\mathrm{Se}$ heteropolar bonds in [SnSe 4$]$ tetrahedral units [19] which built up the main frame of the GSS glass network. The secondary peak located at $260 \mathrm{~cm}^{-1}$ belongs to the vibration mode of the Se-Se bonds in $\mathrm{Se} 8$ rings [30], and it is remarkably broad which indicates that the Se8 rings are highly amorphous and they keep the GSS network in non-crystalline state.

By using the Gaussian decomposition method as illustrated in Fig. 8(a), each Raman spectrum can be decomposed into five Gaussian bands corresponding to vibration mode of structural units in the GSS glass network. According to the results in previous literature, assignment of the five Gaussian bands can be: [ $\left.\mathrm{Se}_{3} \mathrm{Ga}-\mathrm{GaSe}_{3}\right]$ ethane-like units with central frequency at $\sim 173 \mathrm{~cm}^{-1}[31,32]$, symmetric stretching of tetrahedral $\left[\mathrm{SnSe}_{4}\right]$ at $\sim 180 \mathrm{~cm}^{-1}$, symmetric stretching of tetrahedral $\left[\mathrm{GaSe}_{4}\right]$ at $\sim 193$ $\mathrm{cm}^{-1}$ [33], and bending and stretching of [Ses] rings at $\sim 230 \mathrm{~cm}^{-1}$ and $\sim 258 \mathrm{~cm}^{-1}$ [34] respectively, which are tabulated in Table 3. To give the quantized information about the structural changing of the GSS network, proportion of the integral area of each 
structural unit was calculated and plotted as a function of the MCN value as shown in Fig. 8(b). It can be found that only the $\left[\mathrm{Se}_{3} \mathrm{Ga}_{-} \mathrm{GaSe}_{3}\right]$ units exhibit a monotonic evolution (i.e. mere increase) with $\mathrm{MCN}$ value, which parallels the inverse trend of $E_{\text {opg }}$ in Fig. 6. Since value of $E_{\text {opg }}$ has strong positive dependence on strength of

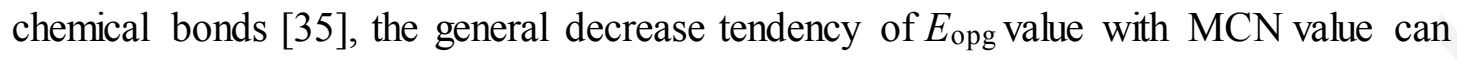
be attributed to the increasing proportion of $\left[\mathrm{Se}_{3} \mathrm{Ga}-\mathrm{GaSe}_{3}\right]$ units which contain the weak bonds, i.e. Ga-Ga homopolar bonds (bonding energy of $193.5 \mathrm{~kJ} / \mathrm{mol}$ [23]) in GSS network. With the same reason, the abnormal $E_{\text {opg }}$ point found at $\mathrm{MCN}=2.55$ (corresponds to sample GSS3) can be attributed to the highest proportion of [ $\left.\mathrm{GaSe}_{4}\right]$ units that consist of Ga-Se heteropolar bonds which are strong bonds (bonding energy of $271.7 \mathrm{~kJ} / \mathrm{mol}$ ) in GSS network energy, as can be observed in Fig. 8(b). Furthermore, the proportions of $\left[\mathrm{Se}_{3} \mathrm{Ga}-\mathrm{GaSe}_{3}\right]$ and $\left[\mathrm{GaSe}_{4}\right]$ units exhibit a reversed trend when the $\mathrm{MCN}$ value passes 2.55, indicating that the $\left[\mathrm{GaSe}_{4}\right]$ tetrahedrons have started to agglomerate and cross-link with each other by Ga-Ga homopolar bonds at this threshold MCN, and such behavior can be regarded as medium-range interactions [36], which resulted in reduction of the total bond energy but enhancement of the network flexibility of the GSS ChGs. Consequently, the conversions of [ $\left.\mathrm{GaSe}_{4}\right]$ tetrahedrons to [ $\left.\mathrm{Se}_{3} \mathrm{Ga}_{-} \mathrm{GaSe}_{3}\right]$ units at $\mathrm{MCN}>2.55$ leads to significant decrease of $E_{\mathrm{opg}}$ value, while the transition temperature $\left(T_{\mathrm{g}}\right)$ and linear refractive index $\left(n_{0}\right)$ (as given in Table 1$)$ of the GSS ChGs exhibits a completely reversed tendency. Besides, it should be noted that proportion of the integral area of $\left[\mathrm{Se}_{3} \mathrm{Ga}-\mathrm{GaSe}_{3}\right]$ at $\mathrm{MCN}=2.69$ has reached $34 \%$ which is larger than that of $\left[\mathrm{Se}_{8}\right](30 \%)$ and $\left[\mathrm{SnSe}_{4}\right](22 \%)$, indicating that the [Se $\left.\mathrm{Se}_{3} \mathrm{Ga}-\mathrm{GaSe}_{3}\right]$ ethane-like units had become the main frame of the GSS glass network after experiencing the maximum level of medium-range interactions. 
On the other hand, as shown in Fig. 8(b), proportion evolution of the [Se8] rings (including both bending and stretching modes) with $\mathrm{MCN}$ value exhibit a completely reversed trend to the tetrahedral $\left[\mathrm{SnSe}_{4}\right]$, indicating they have a competitive relationship in the GSS network. At $\mathrm{MCN}=2.65$, the substitution of [Se8] rings by [SnSe 4$]$ tetrahedral reaches the maximum level, parallels the sudden rising of $E_{\mathrm{g}}$ value at the same $\mathrm{MCN}$ as seen in Fig. 6. Accordingly, the second abnormal $E_{\text {opg }}$ point at $\mathrm{MCN}=2.65$ can be attributed to substitution of the weak Se-Se homopolar bonds (bonding energy of $183.9 \mathrm{~kJ} / \mathrm{mol}$ ) by the strong $\mathrm{Sn}-\mathrm{Se}$ heteropolar bonds (bonding energy of $196.9 \mathrm{~kJ} / \mathrm{mol}$ ) in GSS network with medium-range interactions.

\section{Conclusion}

Glass forming region of arsenic- and germanium-free chalcogenide glasses (ChGs) within a gallium-tin-selenium (Ga-Sn-Se, GSS) ternary system is explored, and the glass forming region is found at Se-rich region in which is composed of $\sim 65-80 \% \mathrm{Se}, \sim 5-15 \% \mathrm{Ga}$, and $\sim 15-25 \% \mathrm{Sn}$. Transition temperature $\left(T_{\mathrm{g}}\right)$ of GSS ChGs varied from 182 to $266{ }^{\circ} \mathrm{C}$ is mainly determined by Ga content, and the GSS ChG with best thermal stability was obtained from composition with lowest Ga content $\left(\mathrm{Ga}_{5} \mathrm{Sn}_{20} \mathrm{Se}_{75}\right)$. GSS ChGs exhibit flat transmittance window range from $\sim 1$ to $20 \mu \mathrm{m}$, and dispersion of the ChGs at 3-5 $\mu \mathrm{m}$ mid-IR window is slightly smaller than the dispersion at 8-12 $\mu \mathrm{m}$ mid-IR window. GSS glass network consist of tetrahedral [SnSe 4$]$, tetrahedral $\left[\mathrm{GaSe}_{4}\right],\left[\mathrm{Se}_{3} \mathrm{Ga}_{-} \mathrm{GaSe}_{3}\right]$ ethane-like units and [Se8] rings, and the structural changing with increase of mean coordinated number $(\mathrm{MCN})$ can be described in term of cross-linking of tetrahedral $\left[\mathrm{GaSe}_{4}\right]$ by $\left[\mathrm{Se}_{3} \mathrm{Ga}_{-} \mathrm{GaSe}_{3}\right]$ ethanelike units and mutual substitution of $\left[\mathrm{Se}_{8}\right]$ rings and tetrahedral $\left[\mathrm{SnSe}_{4}\right]$. Relationship between optical bandgap energy $\left(E_{\mathrm{opg}}\right)$ and $\mathrm{MCN}$ value of the GSS ChGs indicates that medium-range interactions started from GSS glass network with MCN 
$=2.55$, in consistent with the constrained model based on covalent bonding network, and the increasing number of cross-linked [Se $\left.3 \mathrm{Ga}-\mathrm{GaSe}_{3}\right]$ ethane-like units is the origin for such behavior.

\section{Acknowledgements}

This work was partially supported by the National Key Research and Development Program of China (grant no. 2016YFB0303803), National Natural Science Foundation of China (grant no. 61675106) and Natural Science Foundation of Zhejiang Province (grant no. LY19F050005). It was also sponsored by K.C. Wong Magna Fund in Ningbo University.

\section{References}

[1] S. Dai, Y. Wang, X. Peng, P. Zhang, X. Wang, Y. Xu, A review of mid-infrared supercontinuum generation in chalcogenide glass fibers, Appl. Sci. 8 (2018) 707.

[2] Q. Du, Z. Luo, H. Zhong, Y. Zhang, Y. Huang, T. Du, W. Zhang, T. Gu, J. Hu, Chip-scale broadband spectroscopic chemical sensing using an integrated supercontinuum source in a chalcogenide glass waveguide, Photonics Res. 6 (2018) 506-510.

[3] L. Wang, J. Zeng, L. Zhu, D. Yang, Q. Zhang, P. Zhang, X. Wang, S. Dai, All-optical switching in long-period fiber grating with highly nonlinear chalcogenide fibers, Appl. Opt. 57 (2018) 10044-10050.

[4] A. Lemière, F. Désévédavy, P. Mathey, P. Froidevaux, G. Gadret, J.-C. Jules, C. Aquilina, B. Kibler, P. Béjot, F. Billard, O. Faucher, F. Smektala, Mid-infrared supercontinuum generation from 2 to $14 \mu \mathrm{m}$ in arsenic- and antimony-free chalcogenide glass fibers, J. Opt. Soc. Am. B 36 (2019) A183-192. 
[5] H. Li, J. Robertson, Materials selection and mechanism of non-linear conduction in chalcogenide selector devices, Sci. Rep. 9 (2019) 1867.

[6] M. Ghayebloo, M. Rezvani, M. Tavoosi, The relationship between structural and optical properties of Se-Ge-As glasses, Infrared Phy. Technol. 90 (2018) 40-47.

[7] G. Abbady, A.M. Abd-Elnaiem, Thermal stability and crystallization kinetics of $\mathrm{Ge}_{13} \mathrm{In}_{8} \mathrm{Se}_{79}$ chalcogenide glass, Phase Trans. 92 (2019) 667-682.

[8] S. Mishra, P. Lohia, D.K. Dwivedi, Structural and optical properties of $\left(\mathrm{Ge}_{11.5} \mathrm{Se}_{67.5} \mathrm{Te}_{12.5}\right)_{100-\mathrm{x}} \mathrm{Sb}_{\mathrm{x}}(0 \leq \mathrm{x} \leq 30)$ chalcogenide glasses: A material for IR devices, Infrared Phy. Technol. 100 (2019) 109-116.

[9] P. Pradhan, P. Khan, J.R. Aswin, K.V. Adarsh, R. Naik, N. Das, A.K. Panda, Quantification of nonlinear absorption in ternary As-Sb-Se chalcogenide glasses, J. Appl. Phys. 125 (2019) 015105.

[10] E. Zhu, Y. Liu, X. Sun, G. Yin, Q. Jiao, S. Dai, C. Lin, Correlation between thermo-mechanical properties and network structure in $\mathrm{Ge}_{\mathrm{x}} \mathrm{S}_{100-\mathrm{x}}$ chalcogenide glasses, J. Non-Cryst. Solids: X 1 (2019) 100015.

[11] W. Wei, Structural, thermal and optical properties and transition threshold in Ge-Sb-Se glassy system, J. Non-Cryst. Solids 536 (2020) 119991.

[12] Z.H. Zhou, T. Hashimoto, H. Nasu, K. Kamiya, Two-photon absorption and nonlinear refraction of lanthanum sulfide-gallium sulfide glasses, J. Appl. Phys. 84 (1998) 2380-2384.

[13] A. Ravagli, C. Craig, J. Lincoln, D.W. Hewak, Ga-La-S-Se glass for visible and thermal imaging, Adv. Opt. Techn. 6 (2017) 131-136. 
[14] A. Ravagli, C. Craig, K.A. Morgan, I. Zeimpekis, A. Aghajani, E.C. Weatherby, D.W. Hewak, Structural modification of Ga-La-S glass for a new family of chalcogenides, Proc. SPIE 10181 (2017) 101810R.

[15] A. Yang, M. Zhang, L. Li, Y. Wang, B. Zhang, Z. Yang, D. Tang, Ga-Sb-S chalcogenide glasses for mid-infrared applications, J. Am. Ceram. Soc. 99 (2016) $12-15$.

[16] J. Qiu, A. Yang, M. Zhang, L. Li, B. Zhang, D. Tang, Z. Yang, Ga $\mathrm{S}_{3}-\mathrm{Sb}_{2} \mathrm{~S}_{3}-\mathrm{CsI}$ chalcohalide glasses for mid-infrared applications, J. Am. Ceram. Soc. 100 (2017) 5107-5112.

[17] J. Zhou, Q. Du, P. Xu, Y. Zhao, R. Lin, Y. Wu, P. Zhang, W. Zhang, X. Shen, Large nonlinearity and low loss Ge-Sb-Se glass photonic devices in near-infrared, IEEE J. Quantum Electron. 24 (2018) 6101306.

[18] C. Li, P. Guo, W. Huang, W. Zhang, P. Xu, P. Zhang, Reverse-strip-structure $\mathrm{Ge}_{28} \mathrm{Sb}_{12} \mathrm{Se}_{60}$ chalcogenide glass waveguides prepared by micro-trench filling and lift-off, J. Opt. Soc. Am. B. 37 (2020) 82-87.

[19] R. Lin, F. Chen, X. Zhang, Y. Huang, B. Song, S. Dai, X. Zhang, W. Ji, Midinfrared optical properties of chalcogenide glasses within tin-antimony-selenium ternary system, Opt. Express 25 (2017) 25674-25688.

[20] X. Xue, F. Chen, C. Lin, R. Lin, X. Zhang, S. Dai, X. Zhang, W. Ji, Microhardness and optical property of chalcogenide glasses and glass- ceramics of the Sn-Sb-Se ternary system, J. Am. Ceram. Soc. 102 (2018) 2066-2074.

[21] L. Tichý, H. Ticha, Covalent bond approach to the glass-transition temperature of 
chalcogenide glasses, J. Non-Cryst. Solids 189 (1995) 141-146.

[22] G. Saffarini, J.M. Saiter, J. Matthiesen, Thermal stability and percolation threshold of Ge-Se-Fe glasses, Mater. Lett. 61 (2007) 432-436.

[23] A. Giridhar, S. Mahadevan, Chemical ordering in Ge-Ga-Se glasses, J. Non-Cryst. Solids 126 (1990) 161-169.

[24] G. Wang, Q. Nie, X. Shen, X. Wang, F. Chen, S. Dai, T. Xu, Effect of $\mathrm{SnI}_{2}$ on the thermal and optical properties of Ge-Se-Te glasses, Infrared Phy. Technol. 55 (2012) 275-278.

[25] G.E. Snopatin, V.S. Shiryaev, V.G. Plotnichenko, E.M. Dianov, M.F. Churbanov, High-purity chalcogenide glasses for fiber optics, Inorg. Mater. 45 (2009) 1439-1460.

[26] T. Sun, F. Chen, C. Lin, X. Xue, Y. Yang, S. Dai, X. Zhang, W. Ji, Local field effect influenced third-order optical nonlinearity of whole visible transparent chalcogenide glass ceramics, Ceram. Int. 45 (2019) 10840-10844.

[27] A. Srinivasan, K.N. Madhusoodanan, E.S. Gopal, J. Philip, Observation of a threshold behavior in the optical band gap and thermal diffusivity of Ge-Sb-Se glasses, Phys. Rev. B 45 (1992) 8112-8115.

[28] M.M.A. Imran, O.A. Lafi, Glass transition kinetics and optical band gap in $\mathrm{Se}_{85-\mathrm{x}} \mathrm{Sb}_{15} \mathrm{Sn}_{\mathrm{x}}(\mathrm{x}=10,11,12.5$, and 13) chalcogenide glasses, Mater. Chem. Phys. 129 (2011) 1201-1206.

[29] K. Tanaka, Structural phase transitions in chalcogenide glasses, Phys. Rev. B 39 (1989) 1270-1279.

[30] B. Qiao, F. Chen, Y. Huang, R. Lin, X. Zhang, S. Dai, X. Shen, Q. Nie, X. Zhang, 
Correlating structure with threshold behavior of chalcogenide glasses within Ge-Sn-Se ternary system, J. Am. Ceram. Soc. 100 (2017) 108-114.

[31] G. Tang, C. Liu, Z. Yang, L. Luo, W. Chen, Micro-structural studies of $\mathrm{GeSe}_{2}-\mathrm{Ga}_{2} \mathrm{Se}_{3}-\mathrm{MX}\left(\mathrm{MX}=\mathrm{CsI}\right.$ and $\left.\mathrm{PbI}_{2}\right)$ glasses using Raman spectra, J. Non-Cryst. Solids 355 (2009) 1585-1589.

[32] G. Tang, Z. Yang, L. Luo, W. Chen, Preparation and properties of $\mathrm{GeSe}_{2}-\mathrm{Ga}_{2} \mathrm{Se}_{3}-\mathrm{KBr}$ new chalcohalide glasses, J. Alloys Compd. 459 (2008) 472-476.

[33] I. Pethes, R. Chahal, V. Nazabal, C. Prestipino, A. Trapananti, C. Pantalei, B. Beuneu, B. Bureau, P. Jóvári, Short range order in Ge-Ga-Se glasses, J. Alloys Compd. $651(2015) 578-584$.

[34] A.B. Adam, Infrared and Raman studies on $\mathrm{Sn}_{\mathrm{x}}-\mathrm{Sb}_{5}-\mathrm{Se}_{95-\mathrm{x}}$ chalcogenide glasses, J. King Saud Univ. Sci. 21 (2009) 93-97.

[35] G. Saffarini, J.M. Saiter, H. Schmitt, The composition dependence of the optical band gap in Ge-Se-In thin films, Opt. Mater. 29 (2007) 1143-1147.

[36] W. Wei, R. Wang, X. Shen, L. Fang, D. Luther, Barry, Correlation between structural and physical properties in Ge-Sb-Se glasses, J. Phy. Chem. C 117 (2013) 16571-16576. 


\section{Tables}

Table 1 Compositions, characteristic temperatures and other physical parameters of the GSS ChGs as well as the ChGs in previous reports for references.

\begin{tabular}{|c|c|c|c|c|c|c|c|c|c|c|}
\hline \multirow[t]{2}{*}{ Label } & \multirow{2}{*}{$\begin{array}{l}\text { Nominal } \\
\text { composiuvin }\end{array}$} & \multicolumn{3}{|c|}{$\begin{array}{c}\text { Measured } \\
\text { Composition }( \pm 1 \%)\end{array}$} & \multirow{2}{*}{$\begin{array}{l}T_{\mathrm{g}} \\
\left({ }^{\circ} \mathrm{C}\right) \\
\pm 1\end{array}$} & \multirow[t]{2}{*}{$\begin{array}{c}T_{\mathbf{x}} \\
\left({ }^{\circ} \mathrm{C}\right)\end{array}$} & \multirow{2}{*}{$\begin{array}{c}\Delta T \\
\left({ }^{\circ} \mathrm{C}\right) \\
\quad \pm 1\end{array}$} & \multicolumn{2}{|c|}{$\begin{array}{c}d \\
\left(\mathrm{~g} / \mathrm{cm}^{3}\right)\end{array}$} & MCN \\
\hline & & $\mathrm{Ga}$ & & $\mathrm{Se}$ & & & & \pm 2 & \pm 0.001 & \\
\hline GSS1 & $\mathrm{Ga}_{5} \mathrm{Sn}_{20} \mathrm{Se}_{75}$ & & 4.23 & 16.75 & 79.02 & 183 & 329 & 146 & 4.706 & 2.42 \\
\hline GSS2 & $\mathrm{Ga}_{10} \mathrm{Sn}_{16} \mathrm{Se}_{74}$ & & 10.78 & 14.48 & 74.74 & 182 & 277 & 95 & 4.672 & 2.51 \\
\hline GSS3 & $\mathrm{Ga}_{10} \mathrm{Sn}_{17.5} \mathrm{Se}_{72.5}$ & & 11.36 & 15.99 & 72.65 & 185 & 283 & 98 & 4.699 & 2.55 \\
\hline GSS4 & $\mathrm{Ga}_{10} \mathrm{Sn}_{18.5} \mathrm{Se}_{71.5}$ & & 12.56 & 16.98 & 70.46 & 196 & 295 & 99 & 4.725 & 2.59 \\
\hline GSS5 & $\mathrm{Ga}_{10} \mathrm{Sn}_{20} \mathrm{Se}_{70}$ & & 12.77 & 19.73 & 67.50 & 254 & 327 & 73 & 4.810 & 2.65 \\
\hline GSS6 & $\mathrm{Ga}_{10} \mathrm{Sn}_{22.5} \mathrm{Se}_{67.5}$ & & 11.07 & 20.83 & 68.10 & 255 & 336 & 81 & 4.797 & 2.64 \\
\hline GSS7 & $\mathrm{Ga}_{15} \mathrm{Sn}_{20} \mathrm{Se}_{65}$ & & 16.11 & 18.21 & 65.68 & 266 & 319 & 53 & 4.815 & 2.69 \\
\hline \multicolumn{5}{|c|}{$\mathrm{Ge}_{20} \mathrm{Sb}_{15} \mathrm{Se}_{65}$} & 285 & - & - & \multicolumn{2}{|c|}{4.72} & 2.55 \\
\hline \multicolumn{5}{|c|}{$\mathrm{As}_{40} \mathrm{Se}_{60}$} & 182 & - & - & \multicolumn{2}{|c|}{4.63} & 2.40 \\
\hline \multicolumn{5}{|c|}{$\mathrm{Ga}_{9} \mathrm{Sb}_{31} \mathrm{~S}_{60}[15]$} & 244 & 342 & 98 & 4.0 & & 2.49 \\
\hline
\end{tabular}


Table 2 Optical parameters of the GSS ChGs as well as the ChGs in previous reports for reference.

\begin{tabular}{|c|c|c|c|c|c|c|c|c|c|}
\hline \multirow{2}{*}{ Sample } & \multirow{2}{*}{$\begin{array}{l}E_{\text {opg }} \\
(\mathrm{eV})\end{array}$} & \multicolumn{6}{|c|}{$n_{0}( \pm 0.005)$} & \multirow{2}{*}{$\begin{array}{c}3.5 \\
( \pm 1 \%)\end{array}$} & \multirow{2}{*}{$\begin{array}{c}v_{8-12} \\
( \pm 1 \%)\end{array}$} \\
\hline & & 3 um & $4 \mathrm{um}$ & 5 um & 8 um & $10 \mathrm{um}$ & $12 \mu \mathrm{m}$ & & \\
\hline & & 2.486 & 2.480 & 2.476 & 2.469 & 2.464 & 2.459 & & \\
\hline GSS 1 & 1.62 & & & & & & & 148.00 & 146.40 \\
\hline & & 2.483 & 2.477 & 2.474 & 2.467 & 2.463 & 2.457 & & \\
\hline GSS2 & 1.61 & & & & & & & 164.11 & 146.30 \\
\hline & & 2.490 & 2.483 & 2.479 & 2.471 & 2.466 & 2.460 & & \\
\hline GSS3 & 1.63 & 2.508 & 2.501 & 2.497 & 2.488 & 2.482 & 2.476 & 134.82 & 133.27 \\
\hline GSS4 & 1.59 & & & & & & & 136.45 & 123.50 \\
\hline & & 2.534 & 2.526 & 2.521 & 2.512 & 2.504 & 2.496 & & \\
\hline GSS5 & 1.58 & & & & & & & 117.38 & 94.00 \\
\hline & & 2.520 & 2.514 & 2.510 & 2.503 & 2.497 & 2.490 & & \\
\hline GSS 6 & 1.56 & 2.531 & 2.523 & 2.518 & 2.508 & 2.501 & 2.493 & 151.40 & 115.15 \\
\hline GSS 7 & 1.53 & & & & & & & 117.15 & 100.07 \\
\hline & & 2.6116 & 2.6058 & 2.6022 & 2.5929 & 2.5858 & 2.5769 & & \\
\hline $\mathrm{Ge}_{20} \mathrm{Sb}_{15} \mathrm{Se}_{65}$ & 1.70 & & & & & & & 170.8 & 99.1 \\
\hline & & 2.8034 & 2.7964 & 2.7926 & 2.7850 & 2.7795 & 2.7737 & & \\
\hline $\mathrm{As}_{40} \mathrm{Se}_{60}$ & 1.63 & & 286 & & & & & 166.3 & 157.5 \\
\hline $\mathrm{Sn}_{10} \mathrm{Sb}_{20} \mathrm{Se}_{70}[19]$ & 1.03 & & & & & & & 93.1 & 96.5 \\
\hline
\end{tabular}

Table 3 Assignments of the decomposed Raman bands to the structural units in GSS glass network.

\begin{tabular}{ll}
\hline Wavenumber $\left.\mathbf{( c m}^{-\mathbf{1}}\right)$ & Assign ments \\
\hline $165 \sim 175$ & {$\left[\mathrm{Se}_{3} \mathrm{Ga}_{-}-\mathrm{GaSe}_{3}\right]$ ethane-like units } \\
$180 \sim 182$ & {$\left[\mathrm{SnSe}_{4}\right]$ tetrahedrons } \\
$188 \sim 194$ & {$\left[\mathrm{GaSe}_{4}\right]$ tetrahedrons } \\
$230 \sim 236$ & {$\left[\mathrm{Se}_{8}\right]$ rings (bending) } \\
258 & {$\left[\mathrm{Se}_{8}\right]$ rings (stretching) }
\end{tabular}




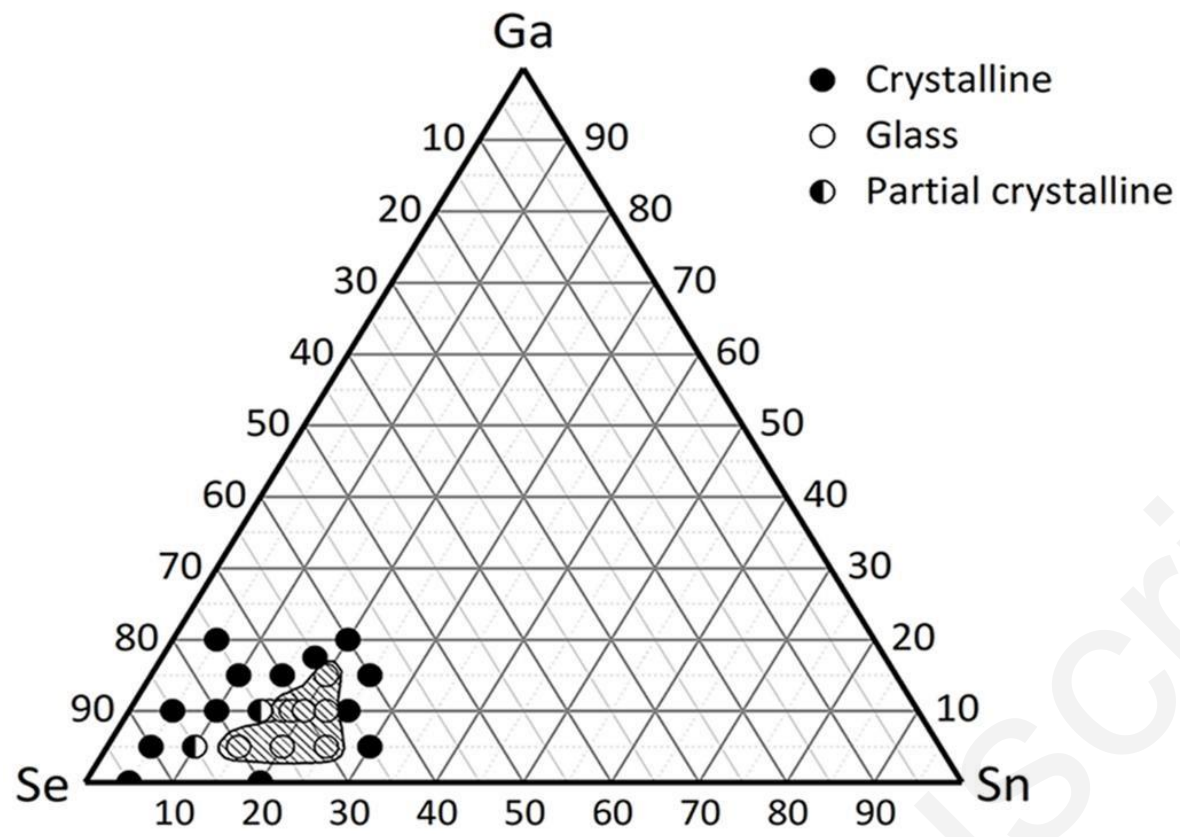

Fig. 1. Glass forming region (in mol\%) of Ga-Sn-Se ternary system, noted as the shadow area. 

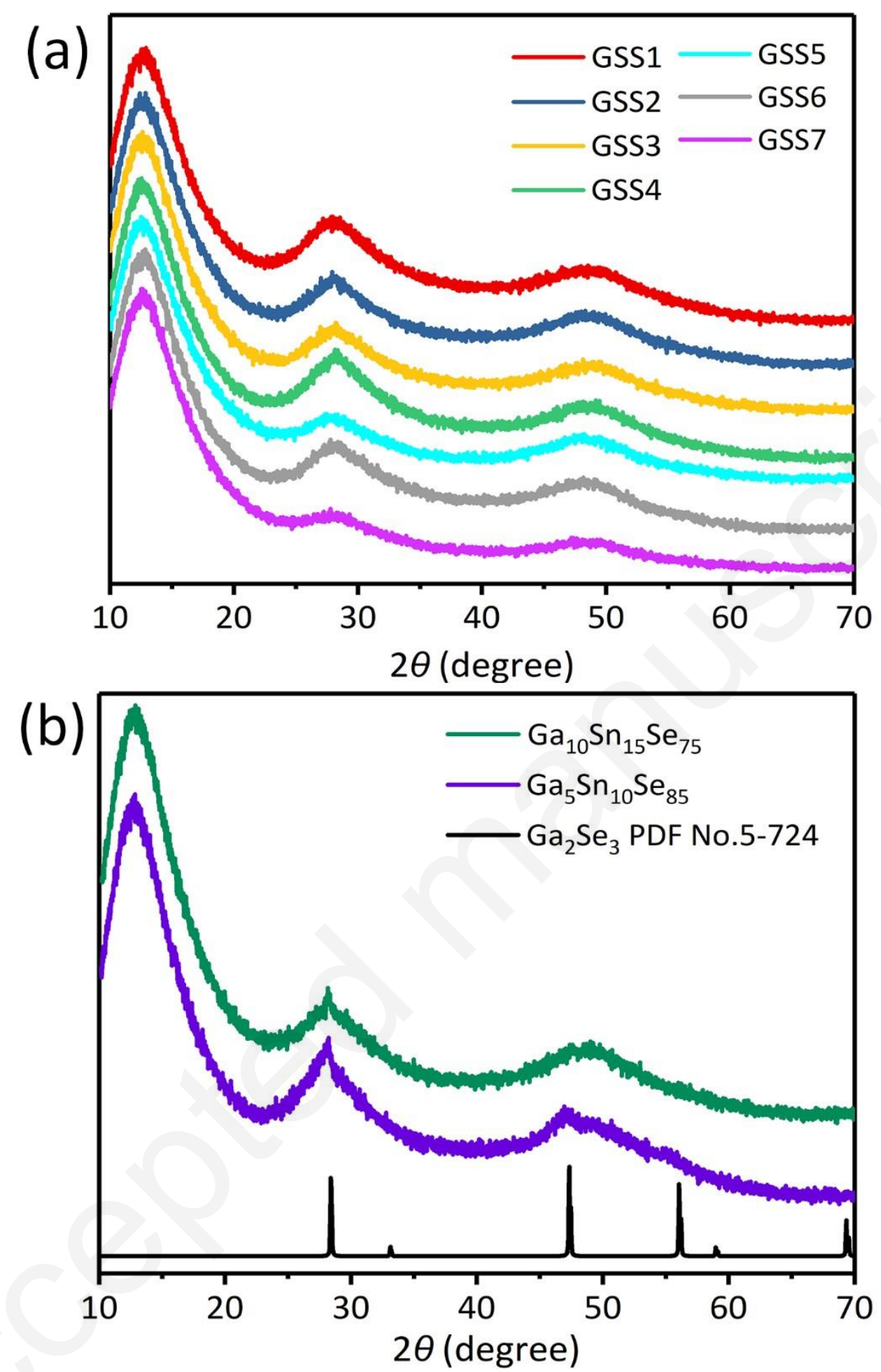

Fig. 2. (a) XRD patterns of GSS samples; (b) identification of the crystal phases in crystallized sample with molar composition of $\mathrm{Ga}_{10} \mathrm{Sn}_{15} \mathrm{Se}_{75}$ and $\mathrm{Ga}_{5} \mathrm{Sn}_{10} \mathrm{Se}_{85}$. 

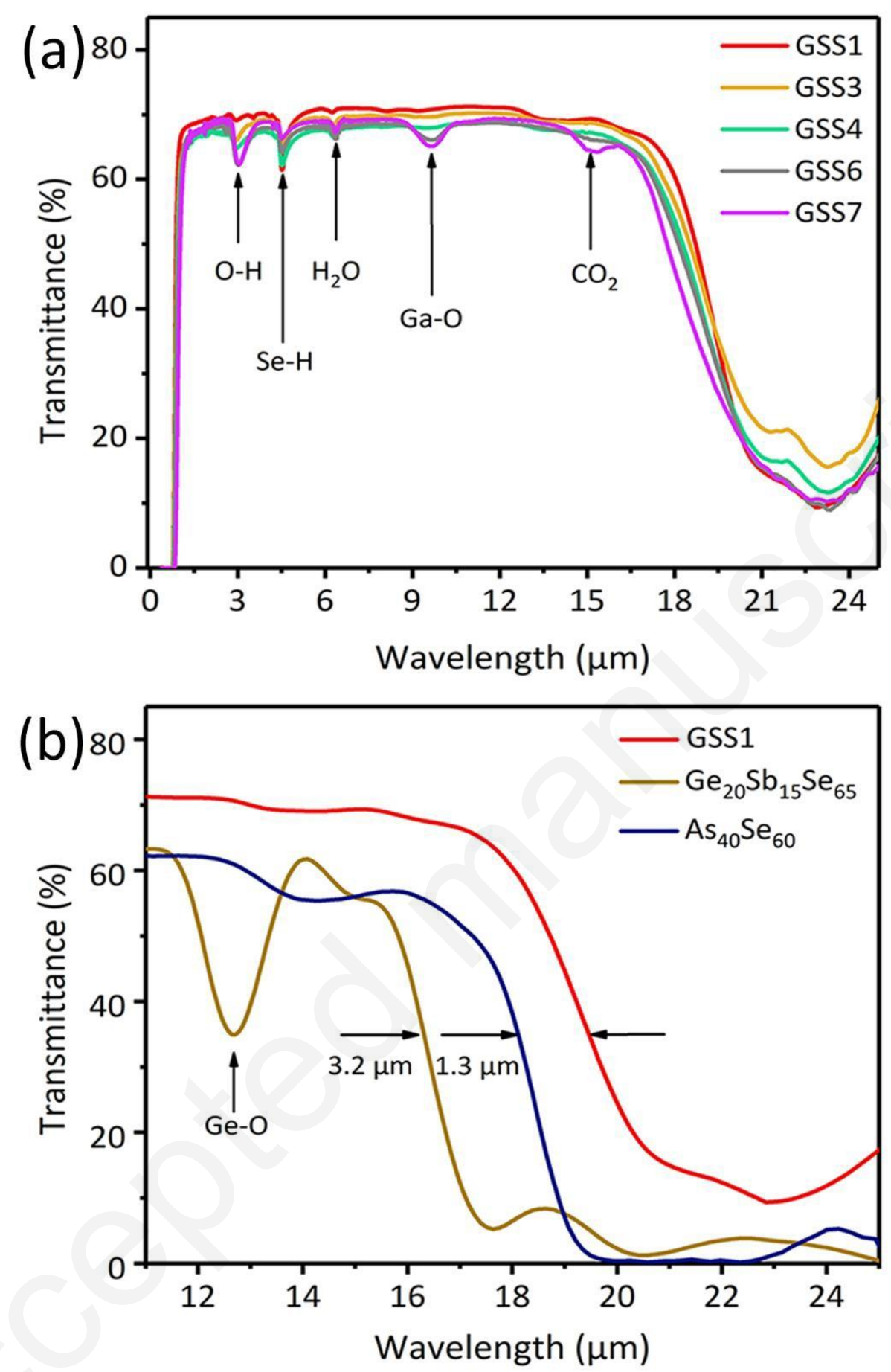

Fig. 3. (a) Full-band transmission spectra of the GSS glasses as well as the notification of impurity absorption; (b) Comparison of FTIR spectra between $\mathrm{Ge}_{20} \mathrm{Sb}_{15} \mathrm{Se}_{65}, \mathrm{As}_{40} \mathrm{Se}_{60}$ ChGs and a GSS sample (GSS1) with the same thickness of 1 $\mathrm{mm}$. 


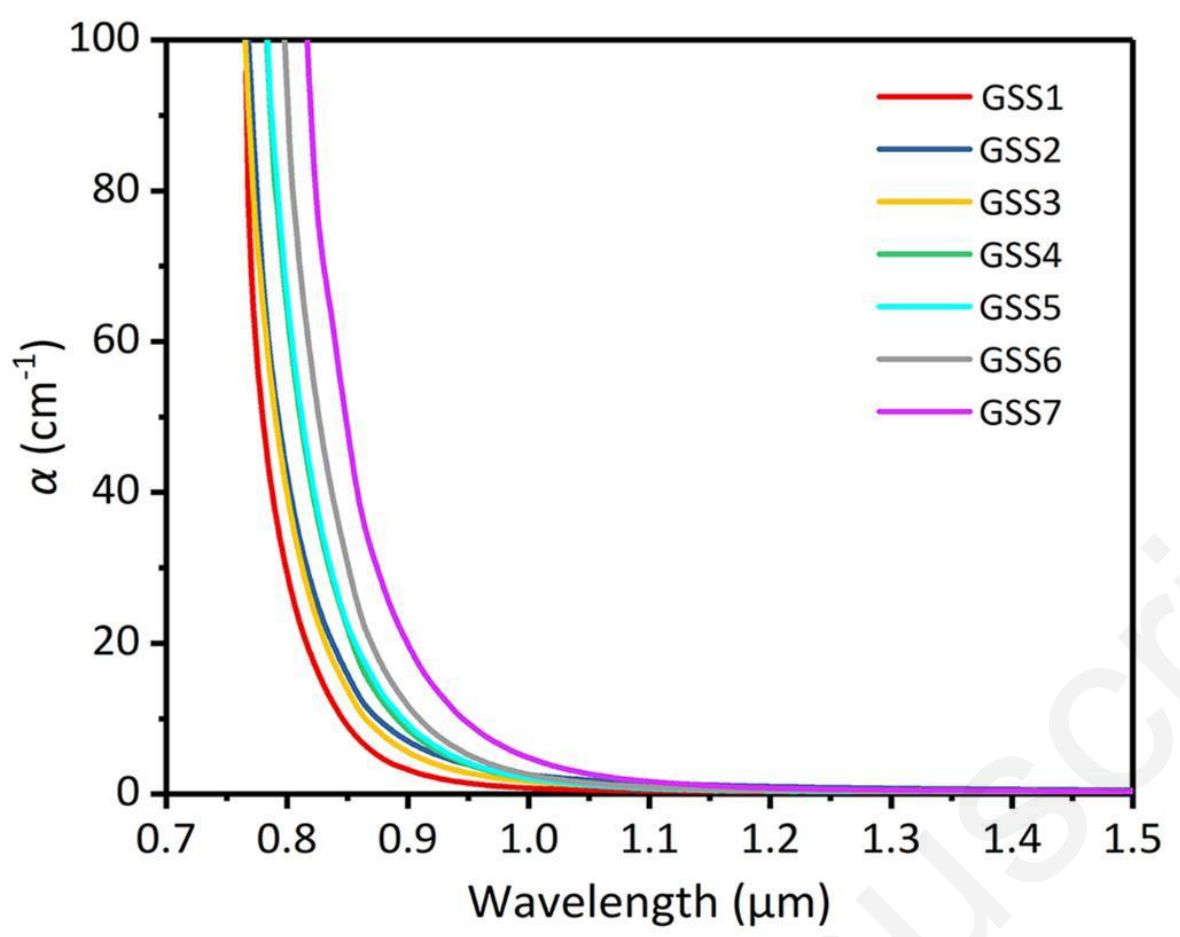

Fig. 4. Near infrared absorption spectra of the GSS ChGs.

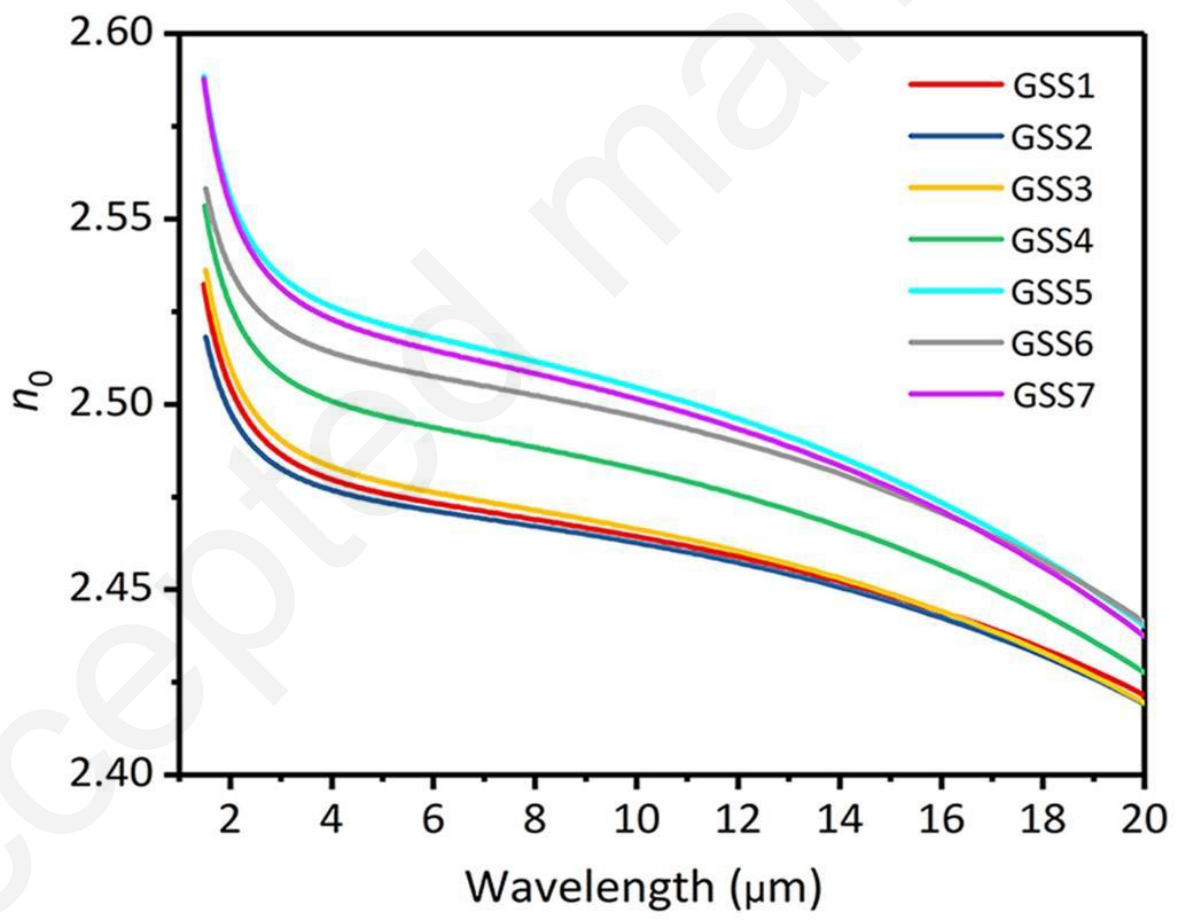

Fig. 5. Linear refractive index $\left(n_{0}\right)$ and its infrared dispersion curves of the GSS ChGs. 


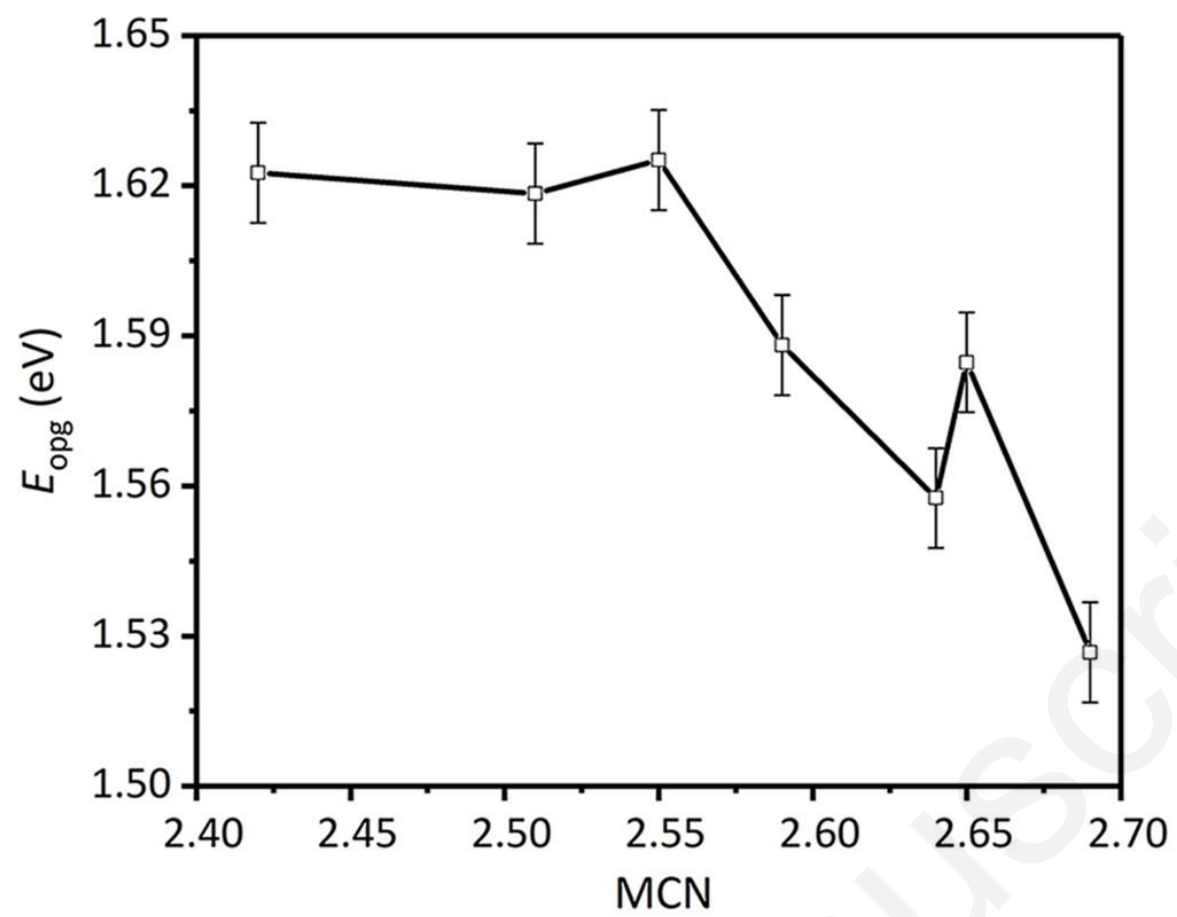

Fig. 6. Optical bandgap energy $\left(E_{\mathrm{opg}}\right)$ versus mean coordination number $(\mathrm{MCN})$ of GSS glasses.

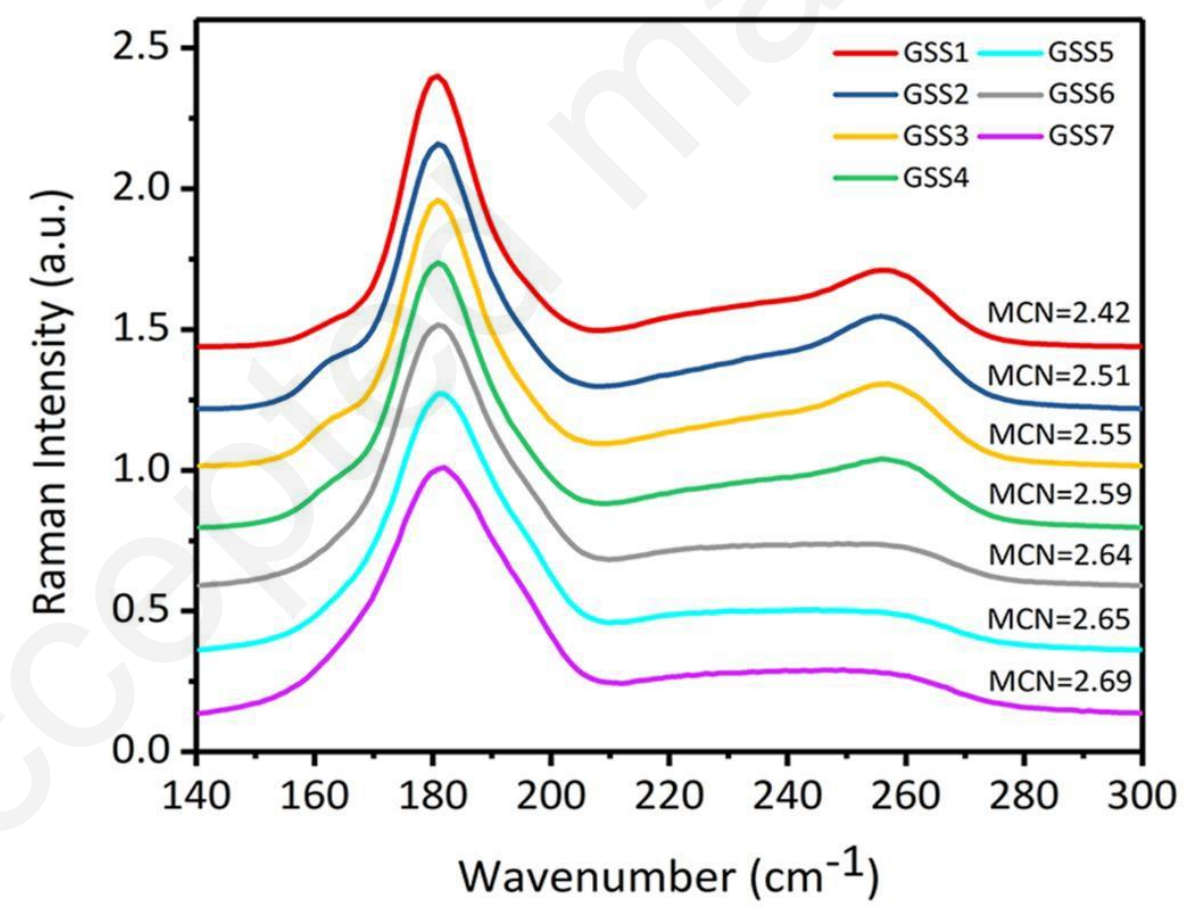

Fig. 7. Normalized Raman spectra of the GSS glasses. 

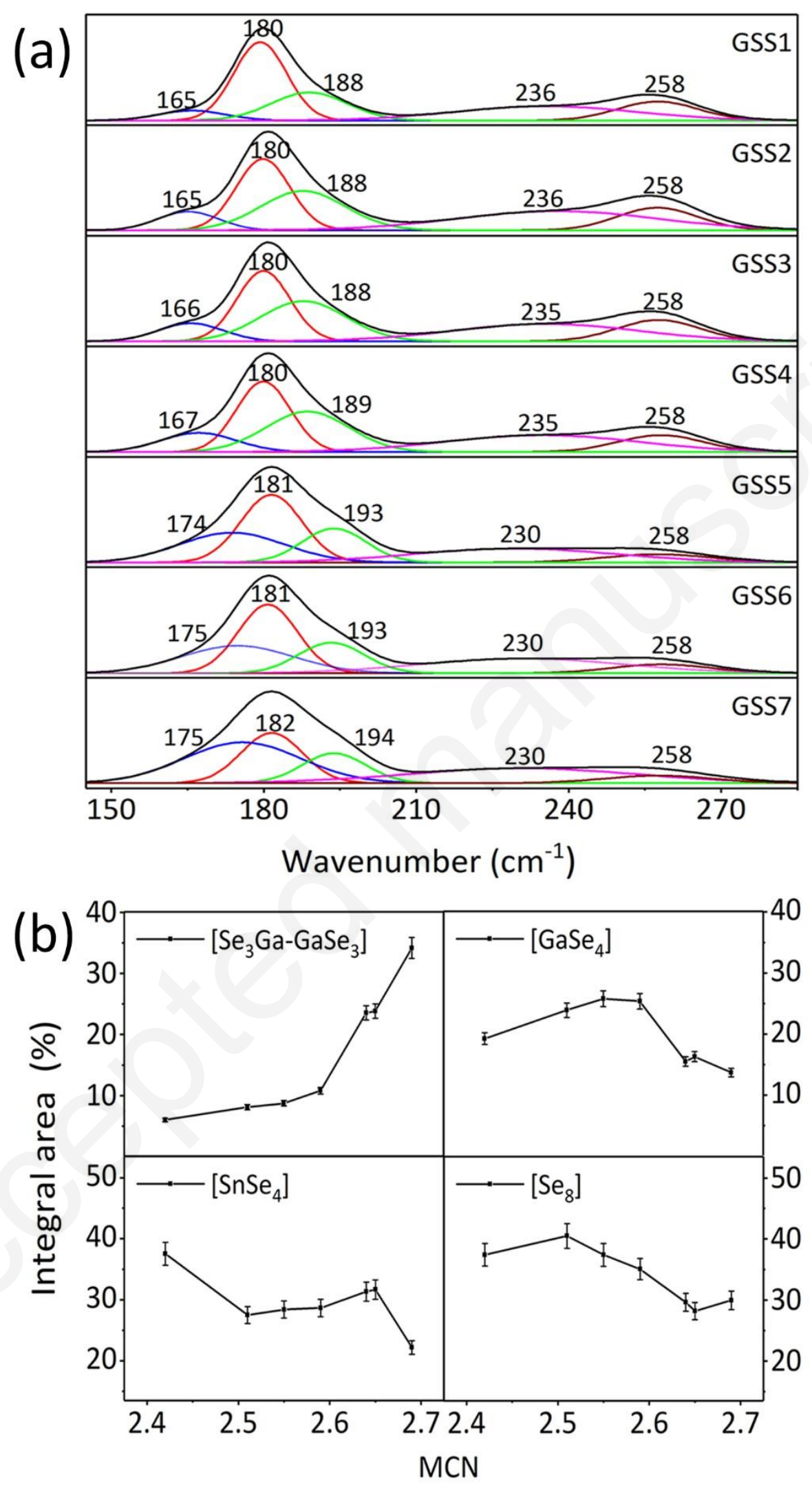

Fig. 8. (a) Decomposition of each Raman spectrum of the GSS ChGs; (b) Proportion of integral area for each Gaussian peak in (a) as a function of MCN. 


\section{Credit Author Statement}

Jiao Zhang, Formal analysis, Writing - Original Draft, Writing - Review \& Editing, Visualization.

Yang Li, Investigation, Methodology.

Chen Zhang, Investigation.

Feifei Chen, Conceptualization, Formal analysis, Writing - Review \& Editing, Funding acquisition, Supervision.

Xianghua Zhang, Conceptualization, Supervision.

Wei Ji, Conceptualization, Supervision. 


\section{Conflict of interest form}

We declare that we have no financial and personal relationships with other people or organizations that can inappropriately influence our work, there is no professional or other personal interest of any nature or kind in any product, service and/or company that could be construed as influencing the position presented in, or the review of, the manuscript entitled. 


\section{Declaration of interests}

$\bigotimes$ The authors declare that they have no known competing financial interests or personal relationships that could have appeared to influence the work reported in this paper.

$\square$ The authors declare the following financial interests/personal relationships which may be considered as potential competing interests: 